\title{
Genome Sequence Data of Three Formae Speciales of Phytophthora vignae Causing Phytophthora Stem Rot on Different Vigna Species
}

\author{
Feifei Sun, ${ }^{1,2}$ Suli Sun, ${ }^{1}$ Wenwu Ye, ${ }^{3}$ Canxing Duan, ${ }^{1}$ Benjin Li, ${ }^{4}$ Weixing Shan, ${ }^{2}$ and \\ Zhendong Zhu' ${ }^{1, \dagger}$ \\ ${ }^{1}$ Institute of Crop Sciences, Chinese Academy of Agricultural Sciences, Beijing, 100081, P. R. China \\ ${ }^{2}$ College of Agronomy of Northwest Agriculture \& Forestry University, Yangling, 712100, P. R. China \\ ${ }^{3}$ Department of Plant Pathology, Nanjing Agricultural University, Nanjing, 210095, P. R. China \\ ${ }^{4}$ Institute of Plant Protection, Fujian Academy of Agricultural Sciences, Fuzhou, 350003, P. R. China
}

\begin{abstract}
Phytophthora vignae is an important oomycete pathogen causing Phytophthora stem rot on some Vigna spp. Three $P$. vignae isolates obtained from mung bean, adzuki bean, and cowpea exhibited high similarities in morphology and physiology but are specialized to infect different hosts. Here, we report the first de novo assembly of the draft genomes of three $P$. vignae isolates, which were performed using the PacBio SMRT Sequel platform. This study will extend the genomic resource available for the Phytophthora genus and provide a good foundation for further research on comparative genomics of Phytophthora spp. and interaction mechanism between hosts and pathogens.
\end{abstract}

\section{Genome Announcement}

Phytophthora stem rot caused by Phytophthora vignae is an important soilborne disease on Vigna crops. The disease was first reported on cowpea (Vigna unguiculata) in Queensland, Australia and the causal agent was identified as $P$. vignae (Purss 1957). P. vignae had a narrow host range and initially was not regarded to infect leguminous plants other than cowpea. In 1978, Kitazawa et al. (1978) identified the pathogen inciting Phytophthora stem rot of adzuki bean ( $V$. angularis) in Hokkaido, Japan as $P$. vignae, and found that it attacks only the three cultivars of adzuki bean used but did not affect another 14 cultivars of other crops tested. Subsequently, Kitazawa et al. (1979) found that the cowpea and adzuki bean isolates attack both cowpea and adzuki bean plants but the $P$. vignae isolates from cowpea were found to be more virulent to cowpea while the $P$. vignae isolates from adzuki bean were more virulent to adzuki bean plants. Tsuchiya et al. (1986) proposed two formae speciales, $P$. vignae f. sp. vignae and $P$. vignae f. sp. adzukicola, for the two organisms on the basis of their specific pathogenicity and host range. Zhu and Wang (2003) found that $P$. vignae f. sp. adzukicola isolates are not only virulent to adzuki bean but also infect individual cowpea and mung bean cultivars by using different inoculation methods. In recent years, $P$. vignae was also identified as the causal agent of stem rot on mung bean ( $V$. radiata) in China. The pathogen showed similar morphology but host range was different from that of $P$. vignae f. sp. vignae and $P$. vignae f. sp. adzukicola. The $P$. vignae isolates from mung bean showed strong virulence to mung bean and relatively weak virulence to adzuki bean but no virulence to cowpea or other legume crops. Hence, these isolates were identified as a new forma specialis of $P$. vignae, $P$. vignae f. sp. mungcola (Sun et al. 2021). To date, the whole-genome sequences of many species in the genus

${ }^{\dagger}$ Corresponding author: Z. Zhu; zhuzhendong@ caas.cn

The author(s) declare no conflict of interest.

Accepted for publication 14 May 2021.
Funding:

This study was supported by the Modern Agro-industry Technology Research System (CARS-08) and the Scientific Innovation Program of the Chinese Academy of Agricultural Sciences.

\section{Keywords}

de novo assembly, field crops, genome resource, oilseeds and legumes, oomycetes 
Table 1. Isolate information and summary statistics of the draft genome assembly for the three formae speciales of Phytophthora vignae

\begin{tabular}{lccc} 
& \multicolumn{2}{c}{ P. vignae isolates } \\
\cline { 2 - 4 } Features & PcV2 & PaV1 & PVMG4 \\
Host & Vigna unguiculata & V. angularis & V. radiata \\
Source & Provided by Prof. Benjin Li & Gannan County, Heilongjiang & Anhui, Mingguang \\
Year & 2017 & 2017 & 2017 \\
Genome size (Mb) & 84.5 & 84.0 & 89.9 \\
Sequenced reads (Gb) & 12.3 & 11.1 & 9.99 \\
Number of contigs & 227 & 203 & 268 \\
Contig N $N_{50}$ & $1.14 \mathrm{Mb}$ & $1.04 \mathrm{Mb}$ & $567.8 \mathrm{~Kb}$ \\
Largest contig (bp) & $3,085,209$ & $2,857,727$ & $3,246,594$ \\
Number of predicted genes & 23,083 & 23,024 & 25,518 \\
Secreted proteins & 1,602 & 1,588 & 1,712 \\
GC content (\%) & 54.9 & 55.0 & 54.8 \\
BUSCO completeness (\%) & 100 & 99 & 98 \\
\hline
\end{tabular}

Phytophthora have been published (Cui et al. 2019; Lee et al. 2020; Srivastava et al. 2020), providing novel insights into the molecular basis of plant disease, such as the identification of secreted RXLR and crinkler (CRN) effector proteins that are associated with plant-pathogen interactions (Kong et al. 2017; Stam et al. 2013; Tyler et al. 2006). Here, we sequenced and assembled the genomes of three isolates which represented different formae speciales of $P$. vignae, and expect that this will provide fundamental understanding of the genetic mechanism underlying host-pathogen interactions and reveal the evolutionary relationship among the three formae speciales of $P$. vignae.

Three isolates ( $P$. vignae f. sp. mungcola PVMG4, $P$. vignae f. sp. adzukicola PaV1, and $P$. vignae f. sp. vignae PcV2) were grown on V8 juice agar at $25^{\circ} \mathrm{C}$ under a 12 -h photoperiod for 7 to 10 days. Genomic DNA of the three isolates was extracted from mycelia using the cetyltrimethylammonium bromide method (Doyle 1987). The quality of the extracted DNA was tested using a NanoDrop One UV-Vis spectrophotometer (Thermo Fisher Scientific) and Qubit 3.0 Fluorometer (Invitrogen). Genome size of the three isolates was evaluated by paired-end sequencing with a 400-bp library on the Illumina Hiseq Xten platform. According to $17 \mathrm{~K}$-mer statistics, $P$. vignae f. sp. mungcola isolate PVMG4 was estimated to be $106.6 \mathrm{Mb}$, with a heterozygosity of $0.5 \%$. The genome size of the other two isolates, PaV1 and PcV2, was assessed to be 100.5 and $98.0 \mathrm{Mb}$, respectively. The GC-Depth showed that there were few contaminants in the sequenced reads. Moreover, preassembly of genomes of the three isolates by SOAPdenovo showed a lower integrity; thus, we used third-generation sequencing technology instead. For PacBio sequencing, SMRTbell libraries of a 20-kb-long insert fragment were constructed and sequenced on the PacBio Sequel platform (Pacific Biosciences) with one singlemolecule real-time (SMRT) cell by Wuhan NextOmics (https://www.grandomics.com/) (Wuhan, China).

After filtering out the reads with low quality and trimming adapter sequences, clean subreads of $12.3,11.1$, and $9.99 \mathrm{~Gb}$ for PcV2, PaV1, and PVMG4, respectively, were used for de novo assembly by the FALCON v1.8.7 assembler with appropriate parameters (Chin et al. 2016) (Table 1). Arrow correction of the assembly results was performed using blasr 4.0 and bwa mem 0.7.12-r1039 with default parameters. The final assembled genome size of the three isolates ranged from 84.0 to $89.9 \mathrm{Mb}$, containing 203 to 268 contigs (Table 1). The contig $\mathrm{N}_{50}$ for PVMG4, PaV1, and PcV2 was 567,800 bp, $1.04 \mathrm{Mb}$, and 1.14 Mb, respectively. Genome coverages were all more than $100 \times$ and GC content of the three isolates was approximately $55 \%$ (Table 1). The completeness of the draft genome assemblies was evaluated using Benchmarking Universal Single-Copy Orthologs (BUSCO) v 4.1.4 (Simão et al. 2015) by comparing 100 eukaryotic single-copy orthologs (SCOs) in the Stramenopiles dataset. The results showed that 100,99 , and $94 \%$ of SCO genes were found as a complete single copy for PcV2, PaV1, and PVMG4, respectively; 0,0 , and $4 \%$ were completely duplicated; 0,1 , and $2 \%$ were missing; and none were fragmented in all three genomes. The gene prediction was performed by Augustus 2.4 (Stanke and Waack 2003), GlimmHmm 3.0.4 (Majoros et al. 2004), GeneWise 2.4.1 (Birney and Durbin 2000), and PASA 2.3.3 (Haas et al. 2008), and the final gene sets were obtained by integrating all the above methods using EVM 1.1.1 (Haas et al. 2008) and, meanwhile, removing transposable elements using TransposonPSI (http://transposonpsi. 
sourceforge.net) (Yagi et al. 2014). In total, 23,083, 23,024, and 25,518 genes were predicted in PcV2, PaV1, and PVMG4, respectively and the mean length of all coding sequences was approximately $1,400 \mathrm{bp}$. Of these genes, the annotated functional genes accounted for $97 \%$ in the three $P$. vignae isolates predicted by InterProScan v5.2-45.0. The gene numbers were similar to those of $P$. sojae in clade 7 (Sharma et al. 2015). To identify the secreted proteins, we used the software SignalP v5.0 to predict signal peptide and, meanwhile, removed the proteins with transmembrane domains predicted by TMHMM v2.0. In total, 1,712, 1,602, and 1,588 secreted proteins were predicted in the genomes of PVMG4, PcV2, and PaV1, respectively, which may contain conserved and virulence-related RXLR and CRN effectors.

We used OrthoMCL to conduct the gene family analysis in $P$. vignae and 121, 34, and 47 unique families were identified in $P$. vignae f. sp. mungcola PVMG4, $P$. vignae f. sp. adzukicola $\mathrm{PaV} 1$, and $P$. vignae f. sp. vignae PcV2, respectively. Phylogenetic analysis based on SCOs of several sequenced Phytophthora spp. ( $P$. sojae, $P$. infestans, and $P$. parasitica) and these three $P$. vignae isolates (PVMG4, PaV1, and PcV2) showed that $P$. vignae and $P$. sojae were clustered in a group and, at the same time, PVMG4, PaV1, and PcV2 were further divided into two branches. These data, coupled with the information about the plant-pathogen interaction in this study, will help further research on host specificity of the three formae speciales of $P$. vignae from different Vigna spp. (V. unguiculata, V. angularis, and V. radiata).

Data availability. The whole-genome shotgun projects of the three $P$. vignae isolates have been deposited at DNA Data Bank of Japan/European Nucleotide Archive/GenBank under the accession numbers JABJUM000000000 (PcV2), JABJUN000000000 (PaV1), and JABJXB000000000 (PVMG4). The versions described in this study are the first versions (JABJUM010000000, JABJUN010000000, and JABJXB010000000). The PacBio sequencing reads and genome assemblies for all three isolates have been deposited as an NCBI BioProject under the accession number PRJNA626502. All three isolates have been preserved in the Agricultural Culture Collection of China (ACCC) under ACCC39666 (PVMG4), ACCC39677 (PaV1), and ACCC39676 (PcV2) accessions.

\section{Literature Cited}

Birney, E., and Durbin, R. 2000. Using GeneWise in the Drosophila annotation experiment. Genome Res. 10:547-548.

Chin, C. S., Peluso, P., Sedlazeck, F. J., Nattestad, M., Concepcion, G. T., Clum, A., Dunn, C., O'Malley, R., Figueroa-Balderas, R., Morales-Cruz, A., Cramer, G. R., Delledonne, M., Luo, C. Y., Ecker, J. R., Cantu, D., Rank, D. R., and Schatz, M. C. 2016. Phased diploid genome assembly with single-molecule real-time sequencing. Nat. Methods 13:1050-1054.

Cui, C. M., Herlihy, J. H., Bombarely, A., McDowell, J. M., and Haak, D. C. 2019. Draft assembly of Phytophthora capsici from long-read sequencing uncovers complexity. Mol. Plant-Microbe Interact. 32:1559-1563.

Doyle, J. J. 1987. A rapid DNA isolation procedure for small quantities of fresh leaf tissue. Phytochem. Bull. 19:11-15.

Haas, B. J., Salzberg, S. L., Zhu, W., Pertea, M., Allen, J. E., Orvis, J., White, O., Buell, C. R., and Wortman, J. R. 2008. Automated eukaryotic gene structure annotation using EVidenceModeler and the Program to Assemble Spliced Alignments. Genome Biol. 9:R7.

Kitazawa, K., Suzui, T., and Yanagita, K. 1979. Pathogenicity of Phytophthora vignae Purss to adzuki-bean and cowpea. Ann. Phytopathol. Soc. Jpn. 45: 406-408.

Kitazawa, K., Tsuchiya, S., Kodama, F., Kamjaipaio, W., Ogoshi, A., and Ynagita, K. 1978. Phytophthora stem rot of adzuki-bean (Phaseolus angularis) caused by Phytophthora vignae Purss. Ann. Phytopathol. Soc. Jpn. 44:528-531.

Kong, L., Qiu, X., Kang, J., Wang, Y., Chen, H., Huang, J., Qiu, M., Zhao, Y., Kong, G., Ma, Z., Wang, Y., Ye, W., Dong, S., Ma, W., and Wang, Y. 2017. A Phytophthora effector manipulates host histone acetylation and reprograms defense gene expression to promote infection. Curr. Biol. 27:981-991.

Lee, Y., Cho, K. S., Seo, J. H., Sohn, K. H., and Prokchorchik, M. 2020. Improved genome sequence and gene annotation resource for the potato late blight pathogen Phytophthora infestans. Mol. Plant-Microbe Interact. 33:1025-1028.

Majoros, W. H., Pertea, M., and Salzberg, S. L. 2004. TigrScan and GlimmerHMM: Two open source $a b$ initio eukaryotic gene-finders. Bioinformatics 20:28782879.
Purss, G. S. 1957. Stem rot: A disease of cowpeas caused by an undescribed species of Phytophthora. Queensl. Agric. J. 14:125-154.

Sharma, R., Xia, X., Cano, L. M., Evangelisti, E., Kemen, E., Judelson, H., Oome, S., Sambles, C., Hoogen, D. J., Kitner, M., Klein, J., Meijer, H. J. G., Spring, O., Win, J., Zipper, R., Bode, H. B., Govers, F., Kamoun, S., Schornack, S., Studholme, D. J., Ackerveken, G., and Thines, M. 2015. Genome analyses of the sunflower pathogen Plasmopara halstedii provide insights into effector evolution in downy mildews and Phytophthora. BMC Genomics 16:741-763.

Simão, F. A., Waterhouse, R. M., Ioannidis, P., Kriventseva, E. V., and Zdobnov, E. M. 2015. BUSCO: Assessing genome assembly and annotation completeness with single-copy orthologs. Bioinformatics 31:3210-3212.

Srivastava, S. K., Abad, Z. G., Knight, L. M., Zeller, K., Mavrodieva, V., and Nakhla, M. 2020. Draft genome resource for the ex-types of Phytophthora ramorum, $P$. kernoviae, and $P$. melonis, species of regulatory concern, using ultra-long read MinION nanopore sequencing. Mol. Plant-Microbe Interact. 33:794-797.

Stam, R., Jupe, J., Howden, A. J. M., Morris, J. A., Boevink, P. C., Hedley, P. E., and Huitema, E. 2013. Identification and characterization CRN effectors in Phytophthora capsici shows modularity and functional diversity. PLoS One 8: e59517.

Stanke, M., and Waack, S. 2003. Gene prediction with a hidden Markov model and a new intron submodel. Bioinformatics 19:ii215-ii225.

Sun, F., Sun, S., Yang, Y., Zhou, B., Duan, C., Shan, W., and Zhu, Z. 2021. A novel disease of mung bean, Phytophthora stem rot caused by a new forma specialis of Phytophthora vignae. Plant Dis. 105:2160-2168.

Tsuchiya, S., Yanagawa, M., and Ogoshi, A. 1986. Formae speciales differentiation of Phytophthora vignae isolates from cowpea and adzuki bean. Ann. Phytopathol. Soc. Jpn. 52:577-584.

Tyler, B. M., Tripathy, S., Zhang, X., Dehal, P., Jiang, R. H., Aerts, A., Arredondo, F. D., Baxter, L., Bensasson, D., Beynon, J. L., Chapman, J., Damasceno, C. M., Dorrance, A. E., Dou, D., Dickerman, A. W., Dubchak, I. L., Garbelotto, M., Gijzen, M., Gordon, S. G., Govers, F., Grünwald, N. J., Huang, W., Ivors, K. L., Jones, R. W., Kamoun, S., Krampis, K., Lamour, K. H., Lee, M. K., McDonald, W. H., Medina, M., Meijer, H. J., Nordberg, E. K., Maclean, D. J., Ospina-Giraldo, M. D., Morris, P. F., Phuntumart, V., Putnam, N. H., Rash, 
S., Rose, J. K., Sakihama, Y., Salamov, A. A., Savidor, A., Scheuring, C. F., Smith, B. M., Sobral, B. W., Terry, A., Torto-Alalibo, T. A., Win, J., Xu, Z., Zhang, H., Grigoriev, I. V., Rokhsar, D. S., and Boore, J. L. 2006. Phytophthora genome sequences uncover evolutionary origins and mechanisms of pathogenesis. Science 313:1261-1266.

Yagi, M., Kosugi, S., Hirakawa, H., Ohmiya, A., Tanase, K., Harada, T., Kishimoto, K., Nakayama, M., Ichimura, K., Onozaki, T., Yamaguchi, H., Sasaki, N.,
Miyahara, T., Nishizaki, Y., Ozeki, Y., Nakamura, N., Suzuki, T., Tanaka, Y., Sato, S., Shirasawa, K., Isobe, S., Miyamura, Y., Watanabe, A., Nakayama, S., Kohara, M., and Tabata, S. 2014. Sequence analysis of the genome of carnation (Dianthus caryophyllus L). DNA Res. 21:231-241.

Zhu, Z. D., and Wang, X. M. 2003. Identification of pathogen causing Phytophthora stem rot of adzuki bean and screening for resistant germplasm. J. Plant Prot. 30: 290-294. 